\title{
Kahoot aplicada en la evaluación sumativa en un curso de matemática discreta
}

\author{
Kahoot applied in the summary evaluation in a discrete mathematics course
}

\section{Kahoot aplicado na avaliação sumária em um curso discreto de matemática}

\author{
Ricardo Enrique Valles-Pereira ${ }^{1}$ \\ Dorenis Josefina Mota-Villegas ${ }^{2}$
}

Recibido: junio de 2019

Aceptado: noviembre de 2019

Para citar este artículo: Valles-Pereira, R.E.; Mota-Villegas, D. J. (2020). Kahoot aplicada en la evaluación sumativa en un curso de matemática discreta. Revista Científica, 37(1), 67-77. Doi: https://doi.org/10.14483/23448350.15236

\section{Resumen}

En este artículo se describe una experiencia de aula, fruto de investigación, en la que se evaluó a un grupo de estudiantes del curso de Matemática Discreta en un tema fundamental como lo es la teoría de conjuntos y sus operaciones por medio de diagramas de Venn. Esto, mediante la incorporación de una herramienta tecnológica lúdica Ilamada Kahoot, la cual permite crear presentaciones/juegos interactivos en los que pueden participar una diversa cantidad de personas a la vez, desde dos personas hasta un amplio número, a través de dispositivos electrónicos. El objetivo fue despertar en el estudiantado interés por dominar los temas matemáticos y por agilizar la rapidez del pensamiento mental mediante el estímulo competitivo que supone comparar sus conocimientos con sus pares. Como metodología se realizó un estudio de campo sustentado en la revisión bibliográfica de los elementos teóricos de interés, en el que se empleó como muestra aquellos estudiantes que cursan la asignatura de Matemática Discreta afín a carreras de Ingeniería de Sistemas y Computación, en el período 2018-2019, en una universidad particular latinoamericana. Los resultados preliminares arrojaron una aceptación total de Kahoot como herramienta evaluativa y un buen porcentaje de respuestas correctas relativas al tema evaluado con dicha herramienta.

Palabras clave: conjuntos, evaluación, Kahoot. matemática, operaciones.

\begin{abstract}
This article describes a classroom experience, the result of research, in which a group of students of the Discrete Mathematics course was evaluated on a fundamental topic such as set theory and its operations through Venn diagrams. This, by means of the incorporation of a playful technological tool called Kahoot, which allows to create presentations / interactive games in which a diverse number of people can participate at the same time, from two people to a large number, through electronic devices. The objective was to awaken in the student interest in mastering mathematical subjects and in speeding up the speed of mental thinking through the competitive stimulus of comparing their knowledge with their
\end{abstract}


peers. As a methodology, a field study was carried out based on the bibliographic review of the theoretical elements of interest, in which students who study the subject of Discrete Mathematics related to Systems and Computing Engineering careers were used as a sample, in the period 2018 -2019, in a particular Latin American university. The preliminary results showed a total acceptance of Kahoot as an evaluative tool and a good percentage of correct answers related to the subject evaluated with that tool.

Keywords: evaluation, mathematics, sets, operations, Kahoot.

\section{Resumo}

Na presente investigação, descreve-se umaexperiência presencial em que um grupo de alunos do curso de Matemática Discreta é avaliado em um tópico elementar, Teoria dos Conjuntos e suasoperações, por meio de diagramas de venn, incorporando umaferramenta tecnológica chamada Kahoot - ferramenta web que permite: Criar apresentações / jogosinterativos nos quaisum número diversificado de pessoas pode participar ao mesmo tempo (a partir de duaspessoas até um grande número) através de dispositivos eletrônicos. Com objetivo de criar interesse do aluno por temas matemáticos e em acelerar a velocidade do pensamento mental através do estímulo competitivo de comparar seusconhecimentoscomseus pares. Como metodologia, foi realizado umestudo de campo a partir da revisão bibliográfica dos elementos teóricos de interesse, onde foram utilizados os alunos que estudam a disciplina de Matemática Discreta relacionada àsprofissões de Sistemas e Engenharia de Computação, período 2018-2019, em umauniversidade latino-americana particular. Os resultados preliminares mostraramumaaceitação total de Kahoot como umaferramentaavaliativa, apresentouumaporcentagem positivas de respostas relacionadas aoassunto avaliado comessaferramenta.

Palavras-chaves: avaliação, matemática, conjuntos, operações, Kahoot.

\section{Introducción}

En la actualidad la evaluación de los aprendizajes obtenidos en la educación formal se hace de manera sumativa; es decir, se pondera o mide a través de un instrumento (de naturaleza cualitativa o cuantitativa) lo que el estudiante supone saber sobre cierto contenido impartido. Dentro de esa medición existen parámetros que permiten tomar decisiones sobre si el estudiante puede avanzar o no (dependiendo de la calificación obtenida al utilizar cierto instrumento) a siguientes contenidos; en consecuencia, la evaluación es un eje importante del proceso de enseñanza-aprendizaje de ese tipo de educación.

Este proceso evaluativo de carácter sumativo se hace en momentos diferenciados durante la enseñanza de una asignatura, y con los resultados (cuantitativos generalmente) se define si un estudiante aprueba o no dicha asignatura. Esto supone que el alumno está sometido a cierta presión, al tener que sacar una alta, o, al menos, aceptable calificación (dependiendo de la escala empleada y de la mínima calificación aprobatoria) para poder aprobar, centrándose muchas veces a estudiar para aprobar la evaluación y dejando de lado el aprendizaje en sí mismo, que es, o debería ser, la finalidad principal del proceso.

Esto sucede también en diferentes áreas del saber, pero por ser en nuestro caso de estudio el de la matemática, en el cual es común encontrar la práctica de solo estudiar para una evaluación y no para retener el conocimiento a largo plazo(lo que genera repercusiones desfavorables), al observar cómo la naturaleza de un conocimiento que es jerárquico y cronológico como lo son los saberes matemáticos se ven fragmentados y divorciados año tras año, cuando un estudiante no se apodera del conocimiento de manera definitiva y significativa. Adicional a ello, está el hecho de que el estudiante debe enfrentar un momento preciso de evaluación, lo que supone niveles altos de ansiedad, la cual puede ser un aspecto que influencie de manera negativa en dichos resultados.

En ese sentido, si bien es cierto que los programas de educación formal presumen que debe existir de forma obligatoria una evaluación de tipo sumativa que permita posicionar al estudiante en 
aprobado-reprobado dependiendo del resultado y que, aunque se ven intenciones de cambios dentro del campo evaluativo, en nuestras universidades seguirá primando esta forma de "medir" lo aprendido por los estudiantes por un tiempo más. En consecuencia, lo que se propone si bien no es cambiar de forma radical la forma de evaluar, al menos pretende hacer de esta actividad algo agradable y motivante para el estudiante. Por ese motivo se propuso Kahoot como forma evaluativa-alternativa si se quiere verificar o medir el conocimiento adquirido por los estudiantes de un tema matemático determinado como lo es el de conjuntos.

Evaluar a los dicentes con Kahoot pasó a ser una forma diferente de valoración, lo que permitió al estudiantado despertar el espíritu competitivo y motivarlo a expresar lo aprendido mediante respuestas rápidas, sin que eso supusiera un nivel de ansiedad que influyera de forma negativa (apreciación de los estudiantes) en la demostración de tales conocimientos.

\section{Marco teórico}

\section{El juego y la matemática}

En los últimos años, los juegos y el proceso de enseñanza-aprendizaje de la matemática han estado vinculados en numerosas experiencias de aula (Rodríguez, 2017; Parreño, 2017; Ribosa y Durán 2017; Ortiz, 2014; Rodríguez, Alonso y Muñiz, 2014) lo que deja al descubierto la conexión que tiene el proceso lúdico con los aprendizajes matemáticos. Sin embargo, desde hace algunas décadas atrás, didactas de la matemática estudiaban ya la conexión entre la matemática y el juego; tal es el caso de Guzmán (1989), quien afirma que la matemática, al igual que el juego, son actividades humanas similares, en las que la creatividad y el ingenio se fusionan en actividades "placenteras, libres y constructivas".

De esta manera, son ejercicios similares los de aprender las reglas de un nuevo juego y aprender las reglas de una cierta operación matemática. En palabras de Guzmán: "Un juego cualquiera comienza con la introducción de unas cuantas reglas, algunos objetos iniciales, piezas cuya función queda definida por dichas reglas, exactamente del mismo modo que los objetos de una teoría matemática quedan determinados por definición implícita" (Guzmán, 1989, p.62).

Por otra parte, Guzmán (1989) también afirma que posibles "bloqueos intelectuales" del pensamiento matemático puede superarse a partir de situaciones que se presenten en un contexto de "juego", donde el clima es "amigable" a diferencia del ambiente de seriedad y rigurosidad en el que a menudo se enseña dicha ciencia.

Sobre la base de lo anteriormente escrito, lo que se propuso en esta experiencia de aula fue al juego como ente evaluador (Kahoot).A la vez, funge como motivador para que el estudiante exprese lo aprendido sin la ansiedad y el estrés que genera la realización de un instrumento tradicional de evaluación (cuestionario, prueba...), también se motiva al estudiante a pensar de una forma rápida en función de la competitividad que sugiere "ganarle" a sus pares, en función del tiempo de su respuesta, que, además, debe ser acertada.

La plataforma en la que fueron dispuestas las preguntas que conformaron la evaluación mediante el juego fue Kahoot; no obstante, las estructuras de las preguntas incorporadas en la plataforma se realizaron considerando "las estrategias de aprendizaje mediante el componente lúdico" (Sánchez, 2010, p.16) las cuales se definen a continuación:

\section{Ventajas de incorporar el componente lúdico al aula de clases}

- La posición del estudiante es activa y el ambiente de clases es más amigable, lo que genera un nivel de confianza en el estudiante que no se obtiene en ambientes rígidos de enseñanza.

- El nivel de ansiedad, que está usualmente presente y tiende a ser elevado en el estudiante 
durante la realización de una evaluación, disminuye ya que los estudiantes ganan confianza en sí mismo y no temen cometer errores.

- El estudiante se mantiene alerta en todo momento, lo que facilita su disposición para el aprendizaje, elementos de excitación de los sentidos como: la sorpresa, la risa, la sensación de diversión... son elementos que mantienen motivados al estudiante durante el proceso educativo.

- La función del juego en el proceso de enseñanza aprendizaje puede ser variada, ya que este puede hacer el papel introductorio de un contenido de enseñanza, de aprendizaje y también puede fungir de evaluador. Esta última función es precisamente la que se propuso con Kahoot en el presente trabajo, ya que la naturaleza del juego fue la de evaluar los aprendizajes adquiridos en el tema de teoría de conjunto, usando dicha herramienta lúdica.

- Al ser el juego un agente motivador, permite al profesor tener versatilidad al momento de su uso; así mismo, crea en el estudiante habilidades y capacidades cuando se ve obligado a generar estrategias o mecanismos para superar retos, resolver problemas y contestar de forma correcta a los cuestionamientos suscitados durante la realización de la actividad lúdica.

- Desarrolla habilidades sociales, de cooperación y compañerismo, siempre y cuando el docente dirija la actividad lúdica en un clima de respeto y colaboración entre iguales. Con esta actividad, el estudiante puede desarrollar sus capacidades individuales y colectivas al relacionarse con los compañeros para obtener mejores resultados.

- Genera actitudes comunicativas, ya que los estudiantes deben expresar sus conocimientos, destrezas y habilidades en público; teniendo que, en muchas ocasiones, argumentar sus respuestas y consideraciones generales sobre la solución de problemas planteados de forma general.

\section{Aspectos considerados para llevar a cabo el juego como componente evaluativo}

Para obtener resultados positivos o favorables al aplicar el juego como estrategia de enseñanza, aprendizaje o evaluación en el aula, Sánchez (2010) propuso considerar reflexiones, las cuales fueron tomadas en cuenta en la presente experiencia de aula. Dichas reflexiones son las siguientes:

- El juego debe cubrir el objetivo o la finalidad propuesta para la que fue creado. En este caso, Kahoot es una herramienta que se adapta de forma eficaz a los objetivos de evaluación propuesto, ya que acepta imágenes, caracteres especiales y los elementos necesarios para editar, transformar y evaluar temas matemáticos.

- Deben considerarse aspectos propios del estudiantado al que se le realizará la actividad lúdica (edad, intereses, disponibilidad de materiales necesarios para la realización, personalidad, etapa o nivel de aprendizaje esperado) de lo contrario, la actividad no sería atractiva y se perdería el interés y la motivación del estudiantado. Todos estos aspectos fueron considerados al aplicar Kahoot, en el que el principal aspecto técnico fue el uso del teléfono inteligente por parte del estudiantado, para lo cual, nuestra población no tuvo ningún tipo de dificultad, ya que se contaba con el material adecuado; por otra parte, en cuanto a la motivación, el carácter competitivo del juego mantuvo a la audiencia (estudiantes) motivada en todo momento, independientemente de la edad.

- El juego debe suponer ser un reto, pero uno lograble por parte del estudiante; una actividad demasiado sencilla hace que el estudiante pierda el interés y se distraiga de la misma, mientras que una actividad demasiado compleja hace que se frustre y desmotive. En nuestro particular estudio, las preguntas puestas en Kahoot estuvieron acorde a los objetivos, logrando la motivación adecuada y positiva en el estudiante durante la realización de la actividad. 
- Las reglas del juego deben ser claras, presentadas al inicio de la actividad. En estas incluso se deben dar ejemplos previos al juego formal; si un estudiante confunde o no conoce las reglas del juego, tenderá a abandonarlo y, por lo tanto, el mismo se volverá ineficaz. Para cubrir este aspecto se dio a conocer Kahoot antes del inicio de la actividad, sus reglas, su forma de participación y la posible interacción entre los estudiantes; como ventaja se tuvo el fácil manejo de la aplicación y las condiciones tecnológicas óptimas en el recinto universitario para su funcionamiento sin presentar ninguna eventualidad.

\section{Kahoot como herramienta lúdica evaluativa en matemática}

Kahoot es una herramienta disponible de forma gratuita en la web (https://kahoot.com/) que se emplea principalmente para la elaboración de juegos interactivos basados en preguntas en los que pueden participar desde dos hasta un número limitado de personas de forma simultánea. Actualmente, Kahoot ha sido aplicada en el ámbito educativo con gran aceptación por la comunidad en general, lo que se hace evidente en trabajos como los de Rodríguez-Fernández (2017), Moya et al., (2016), Muñoz (2016), Guimares (2015) y Zarzycka (2014). Sin embargo, no se encontraron referencias anteriores de su uso en el campo de la matemática; lo que acrecentó el interés de los investigadores del presente artículo por realizar el estudio de esta herramienta en el aula y de presentar sus resultados en el campo de la didáctica de la matemática al momento del desarrollo de este estudio.

Para comentar brevemente las reglas del juego, mencionaremos que las personas participan en Kahoot mediante el uso de dispositivos electrónicos inteligentes con conexión a internet (computadoras, teléfonos celulares, tableta, entre otros). Previo a la participación el docente debe tener elaborada la encuesta, cuestionario o el banco de preguntas que se aplicará, seleccionando imágenes, textos, figuras u otros si así lo requieres y determinado el tiempo de duración en pantalla de cada pregunta, la cual tendrá varias opciones de respuesta de selección simple o múltiple.

Luego del vencimiento del tiempo de cada pregunta, el docente administrador de la cuenta Kahoot y los participantes podrán ver la respuesta correcta y cuál de los participantes obtuvo las mejores puntuaciones (primer, segundo y tercer lugar). Dichas puntuaciones se logran considerando que se seleccione la respuesta correcta en el menor tiempo posible. Adicionalmente, el administrador de la cuenta puede acceder a datos estadísticos (en formato xls) del rendimiento de cada estudiante, discriminando su participación en cada pregunta, lo que permite hacer un análisis posterior de todos los resultados obtenidos.

Cabe resaltar que cada banco de preguntas o cuestionario elaborado en la cuenta de Kahoot del docente tiene la particularidad de ser reutilizable para otras actividades de la misma índole, o bien ser editable para agregar, modificar o copiar preguntas y cuestionarios completos. Los cuales nos permiten ir ampliando nuestro portafolio de materiales matemáticos en nuestra cuenta de Kahoot.

Los detalles de cómo acceder, crear una cuenta, cómo jugar, compartir, entre otros se pueden responder en el documento Valijas de Herramientas TIC (2016), disponible en línea cuyo enlace es http://valijas.ceibal.edu.uy/storage/app/media/ Guias \%20de \%20uso/Kahoot.pdf,del cual también se extrajo la información anterior.

\section{Metodología}

\section{Tipo y diseño de investigación}

El presente trabajo se corresponde con una experiencia de aula dentro de la cual se enmarca en el tipo de investigación acción donde, sobre el proceso aplicado, se evaluaron resultados. Sin embargo, no se considera la experiencia como un proceso cerrado, al contrario, constantemente se aplica, ajusta y reaplica el proceso, se evalúan y revalúan los resultados que se consideran cambiantes en todo momento. En 
cuanto al diseño, fue de campo, apoyados en material bibliográfico pertinente y actualizado.

\section{Contexto del estudio y sujetos de la investigación}

La experiencia de aula tuvo lugar en la Pontificia Universidad Católica del Ecuador, Quito-Ecuador. En esta se desarrolló la herramienta Kahoot como base de evaluación del contenido matemático sobre la teoría de conjuntos, aplicada a 31 estudiantes de la modalidad presencial (totalidad de la población de estudio, no se aplicaron criterios muestrales) de 41 inscritos en la asignatura de Matemática Discreta (segundo semestre), afines a carreras de Ingeniería en Sistema y Computación en el periodo académico 2018-2019.

\section{Descripción de la experiencia}

En el contexto antes mencionado, una vez impartido el tema de teoría de conjuntos y ejercicios aplicando diagramas de Venn (Fig. 1) se llevó a cabo el cuestionario Kahoot para validar (evaluación sumativa) los conocimientos adquiridos.
El cuestionario aplicado en forma de juego estuvo conformado por 20 preguntas, cada una con cuatro opciones de respuesta, con una duración aproximada para responder de 20 a 30 segundos, dependiendo de la complejidad de cada pregunta, las cuales estuvieron ajustadas al tema de teoría de conjuntos y propiedades, aplicando diagramas de Venn (Fig. 2).

Una vez iniciado el cuestionario, los estudiantes se mostraron prestos y concentrados en dar respuesta de manera óptima y rápida a cada una de las preguntas plasmadas en la pizarra por medio de Kahoot. Logrando, en este particular, obtener toda su atención al trabajo en Kahoot relacionado con el tema de teoría de conjuntos (figuras 3 y 4 ).

Perspectiva de las preguntas en el desarrollo del cuestionario en Kahoot. Realizada a los estudiantes del curso de Matemática Discreta (figura 4).

Una vez realizado el juego se descargaron los resultados, los cuales se pueden ver y editar en formato Excel obteniéndose información general (figura 5) y detallada (figura 6) de las respuestas seleccionadas por los estudiantes en cada pregunta realizada.

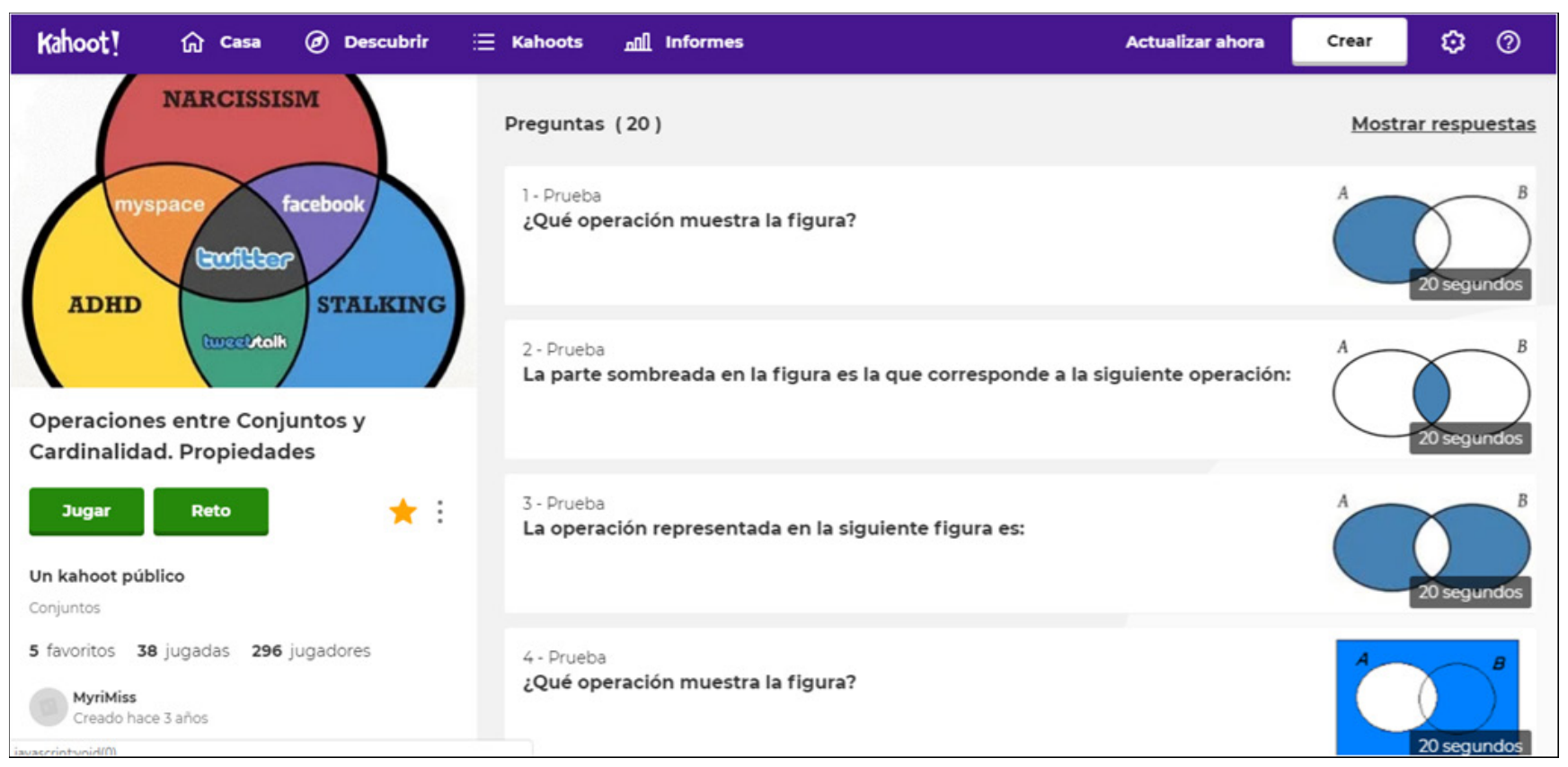

Figura 1. Interfaz de la cuenta Kahoot del docente. 


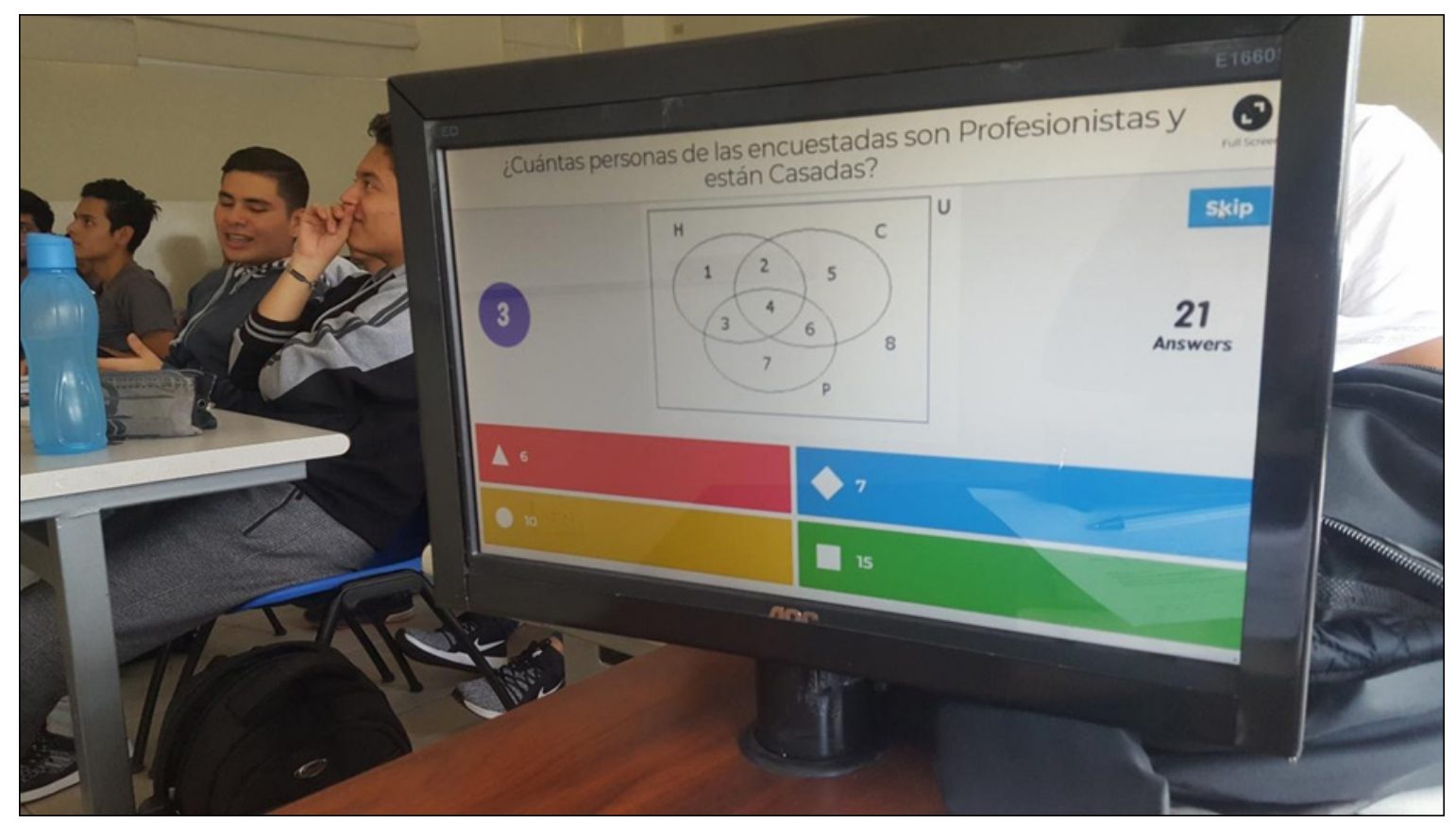

Figura 2. Ejemplo de la estructura de una de las preguntas del cuestionario aplicado sobre teoría de conjuntos en Kahoot.

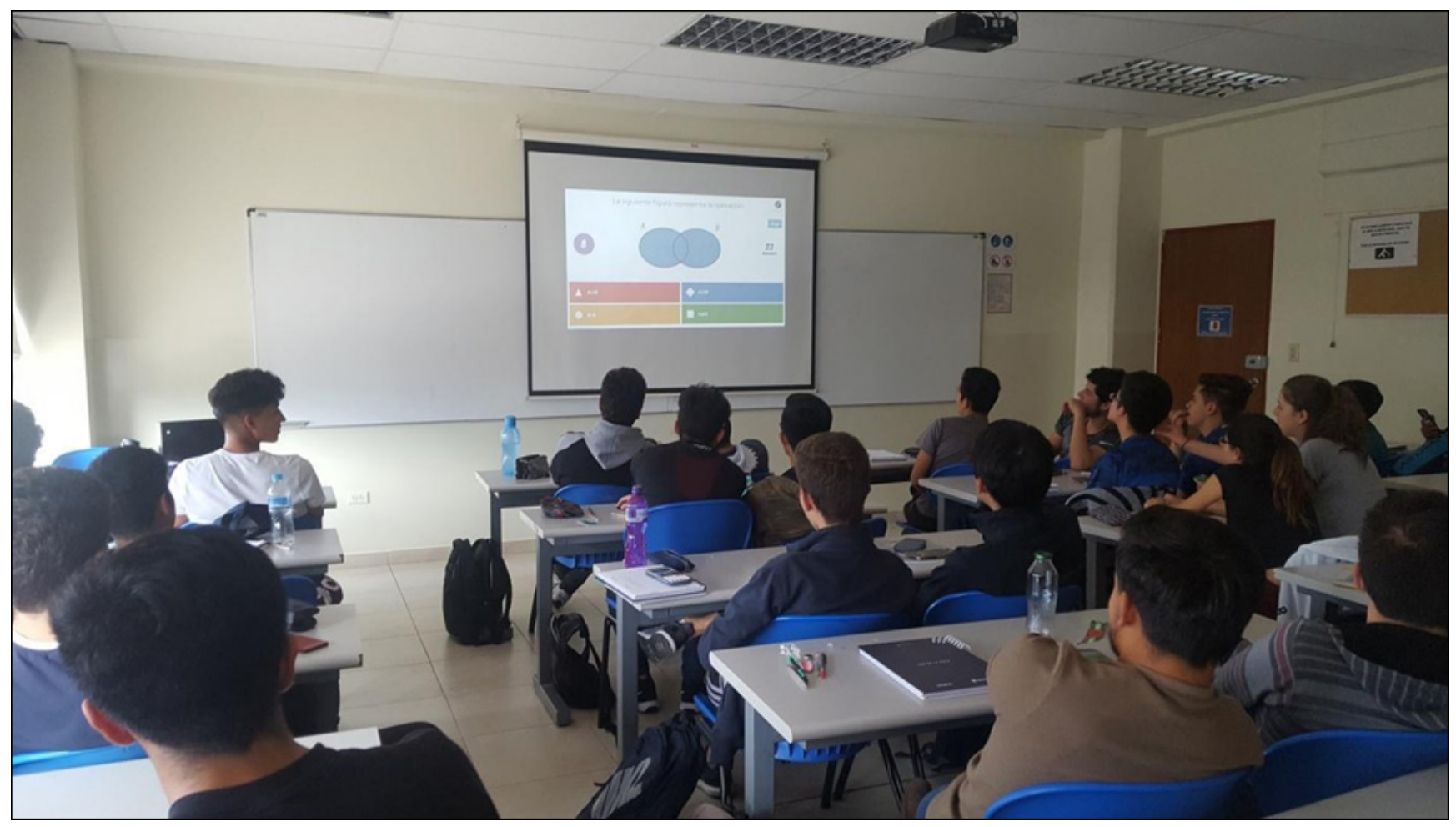

Figura 3. Desarrollo de la actividad por parte de los estudiantes del curso de Matemática Discreta, en el aula, haciendo uso de Kahoot, proyector y pizarra. 


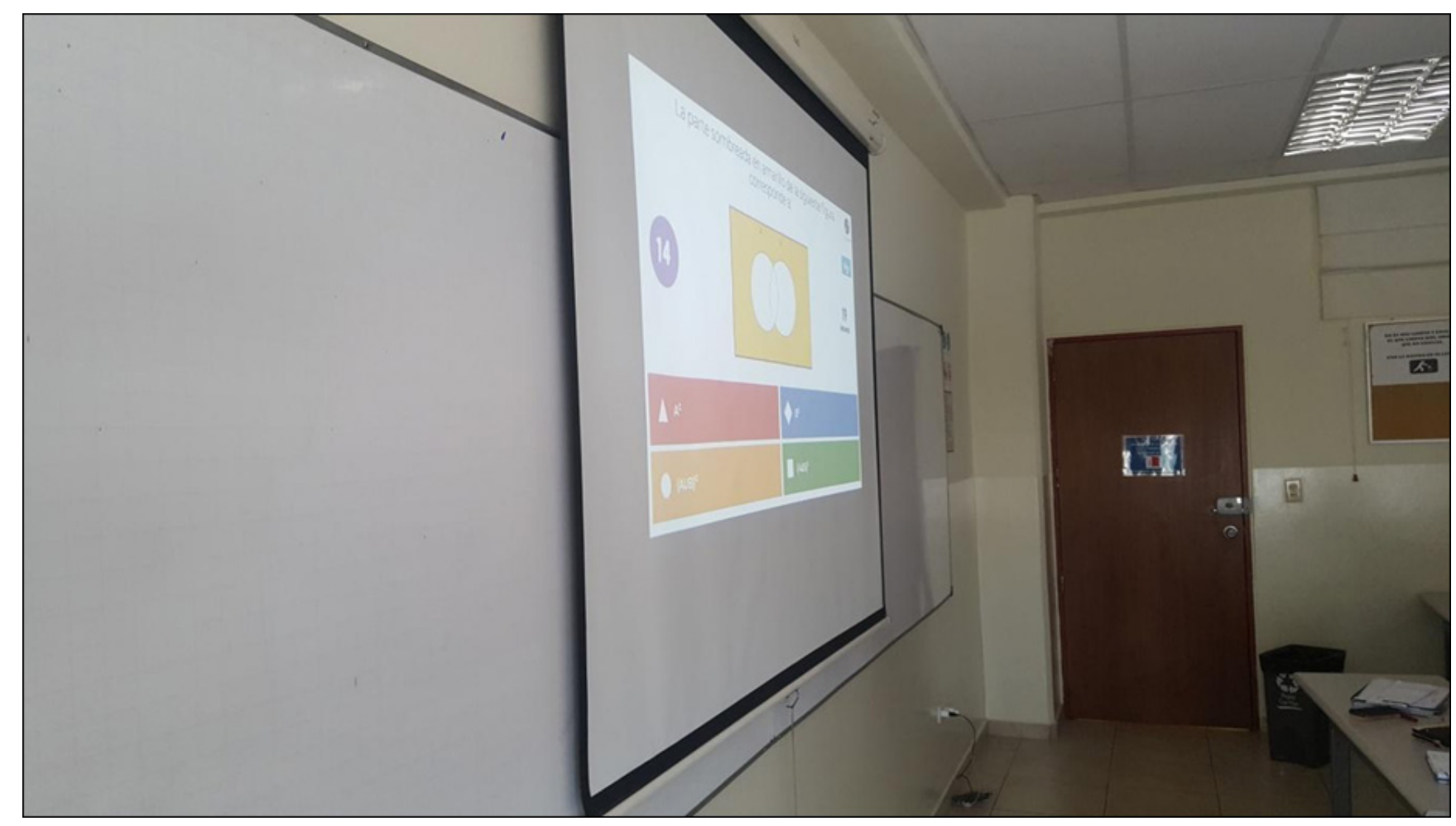

Figura 4. Perspectiva diagonal de la proyección de una de las preguntas en Kahoot, a los estudiantes del curso de Matemática Discreta.

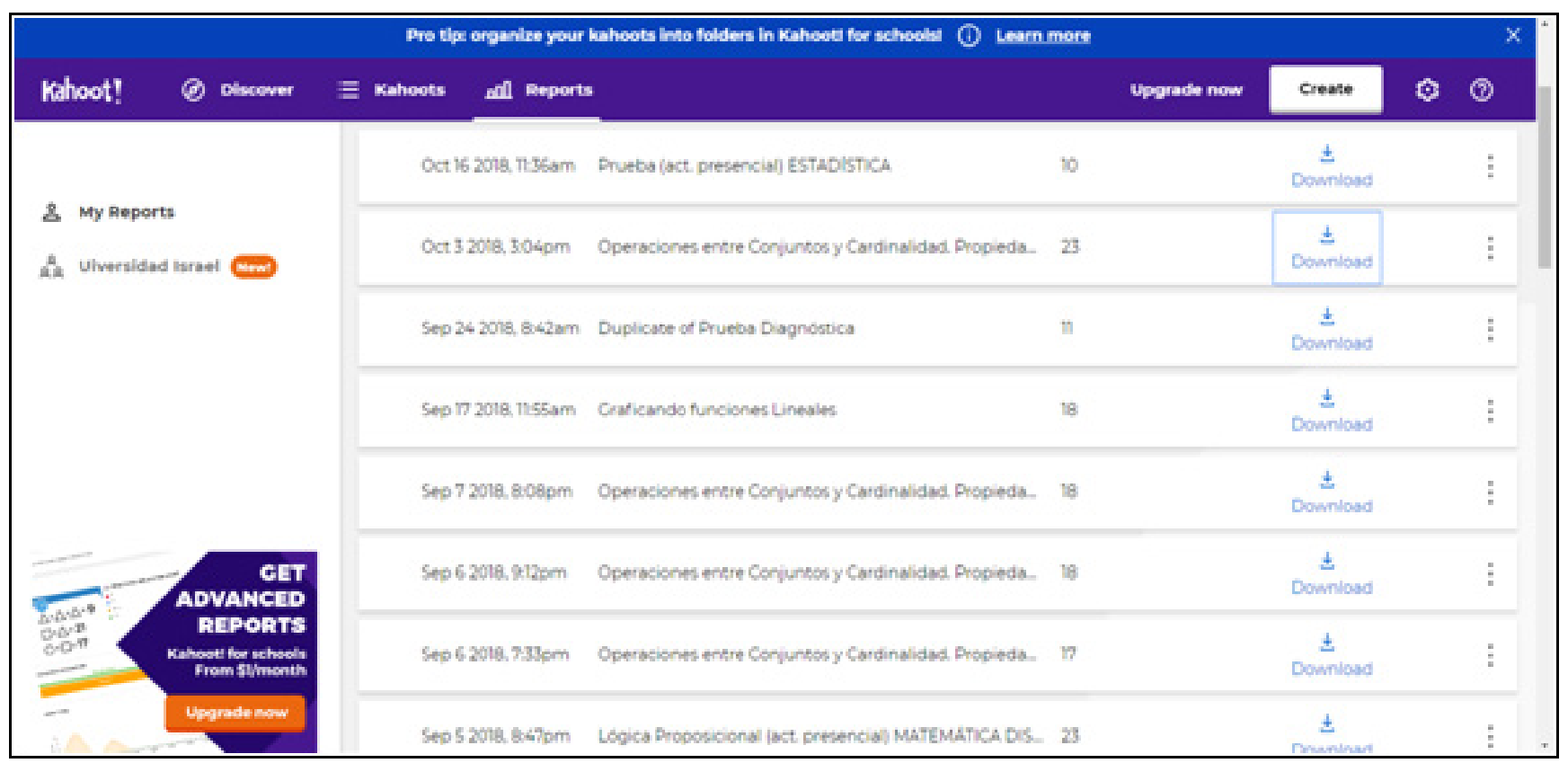

Figura 5. Resultados globales de los diferentes cuestionarios aplicados, descargables en formato.xls, sobre los temas matemáticos en Kahoot. 


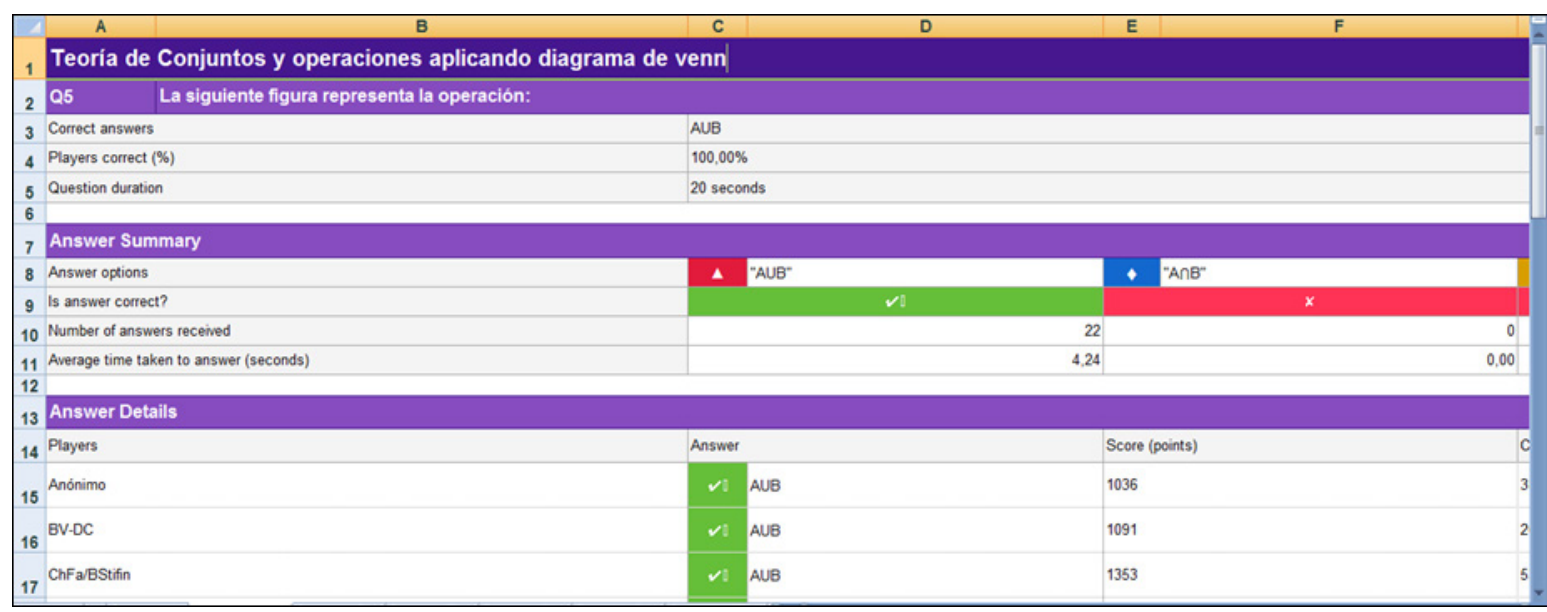

Figura 6. Muestra de los resultados por pregunta en el cuestionario aplicado sobre teoría de conjuntos en Kahoot.

\section{Resultados y análisis}

Los resultados obtenidos de la experiencia fueron analizados desde dos perspectivas. La primera fue desde el punto de vista de la aceptación del estudiante del recurso lúdico Kahoot como medio para ser evaluados en el tema de triángulos, esto se hizo sobre la base de una encuesta estructurada, las respuestas más significativas en la tabla 1 .

Tabla 1. Resultados de la pregunta "¿anteriormente habías usado Kahoot en el aula?".

\begin{tabular}{lrrr}
\hline Respuestas & Uso Kahoot & & Porcentaje $\%$ \\
Sí & 0 & 0 \\
No & 31 & 100 \\
\hline
\end{tabular}

Los resultados de la pregunta expuesta en la tabla 1: el $100 \%$ de los estudiantes contestaron que no habían usado la herramienta Kahook anteriormente, dejando al descubierto la novedad que representó para el estudiantado este tipo de herramientas al aula de clases. Esto sirvió para la motivación correcta inicial necesaria para el desarrollo eficaz de la misma.

Los resultados de la pregunta expuesta en la tabla 2 dan indicios sobre el logro del objetivo del uso como recurso de evaluación de dicha herramienta lúdica; esto debido a la total aceptación o acogida \% del recurso por parte de los estudiantes encuestados.

Tabla 2. ¿Te ha resultado útil la implementación de Kahoot para la evaluación del tema de teoría de conjuntos?

\begin{tabular}{lrrr}
\hline Respuestas & Uso Kahoot & Porcentaje $\%$ & \\
Sí & 31 & & 100 \\
No & 0 & 0 \\
\hline
\end{tabular}

De forma similar, en cuanto los resultados, respondieron los estudiantes a cuestiones parecidas como: ¿sugerirías el uso de Kahoot para otros contenidos o para otras asignaturas?, ¿consideras que tu participación y competitividad en el aula ha mejorado gracias al uso de Kahoot? Obteniéndose resultados favorables al uso de la herramienta en más del $90 \%$ en todos los casos. En forma general, los resultados demuestran la gran receptividad que tiene Kahoot como herramienta de evaluación en la asignatura de Matemática Discreta a los estudiantes de las carreras de Ingeniería de Sistemas y Computación.

Por su parte, la segunda perspectiva de los resultados estuvo orientada a las puntuaciones obtenidas por los estudiantes una vez analizados los resultados de la encuesta puesta en Kahoot en relación con respuestas correctas e incorrectas (Fig. 7). 


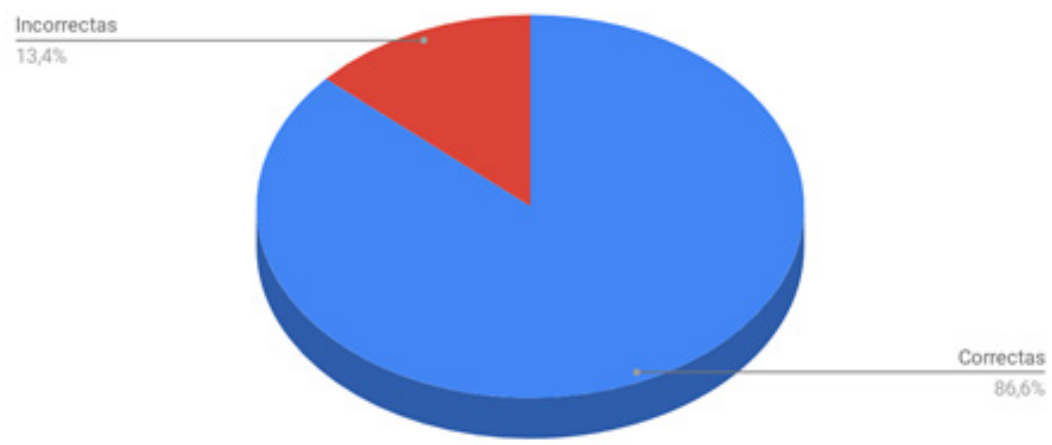

Figura 7. Resultados globales obtenidos por respuestas correctas e incorrectas de acuerdo con el cuestionario aplicado en Kahoot.

Fuente: estadísticos Kahoot.

Se puede observar que un importante porcentaje de estudiantes (87\% aproximadamente) respondieron de forma correcta a las preguntas planteadas en el cuestionario de Kahoot (Fig. 7). Teniendo en cuenta que el contenido se centra en un tema matemático (teoría de conjuntos) es satisfactorio obtener ese porcentaje de aciertos; sin embargo, valdría la pena comparar estos resultados con los de otro grupo similar a los que no se le aplique Kahoot en dicha evaluación, para poder comprobar la eficacia de una forma más objetiva de Kahoot como herramienta evaluadora; en ese sentido, se estima hacer este estudio comparativo en un futuro próximo.

\section{Conclusiones}

Kahoot como herramienta lúdica evaluativa en el área de Matemática parece ser, de entrada, un factor motivante y atractivo que permite mejorar la forma de obtener información objetiva del conocimiento del estudiantado (evaluación sumativa). Esta evita la tensión, el estrés y niveles altos de ansiedad que suponen este tipo de evaluaciones tomadas de forma tradicional (examen escrito). No obstante, en esta primera aproximación de su uso se utilizó como tema las nociones elementales sobre la teoría de conjuntos y aplicaciones con diagramas de Venn, lo que evoca sustancialmente a la memoria del estudiante para realización de las respuestas de forma correcta. En ese sentido, puede afirmarse que construyó a la mejora de la memoria de propiedades y nociones elementales sobre la teoría de conjuntos; pero no nos da referencia sobre procesos matemáticos más complejos, precisamente por la estructura de las preguntas de selección simple .Sin embargo, en futuras aplicaciones podrán plantearse problemas donde intervengan procesos matemáticos para su resolución; en ese caso, serían preguntas en las que se plantee más tiempo de respuesta; la posibilidad queda abierta por el momento.

En todas las preguntas planteadas sobre la valoración de la herramienta Kahoot y de su uso en el aula los estudiantes mostraron resultados favorables al respecto, manifestando estar de acuerdo con el uso de esta herramienta evaluativa y también se hizo manifiesta la aceptación para seguir usándola en el futuro .Un porcentaje importante (más del 85 \%) respondió de forma correcta las preguntas planteadas en Kahoot, sin embargo, habría que plantearles cuestiones o preguntas más complejas para evidenciar el impacto real que dicha herramienta lúdica tiene desde el punto de vista del rendimiento académico.

Para finalizar, se hace evidente la gran motivación que crea en el estudiantado el uso de Kahoot en el aula de clases como herramienta evaluadora en la materia de Matemática Discreta. En definitiva, la alta valoración de los estudiantes hacia el uso de la herramienta la hace un aliado poderoso para evaluar (a priori conocimientos que deben ser memorizado) conocimientos matemáticos 
elementales; esto nos insta a seguir aplicando la herramienta y continuar analizando el impacto de la misma en el proceso educativo. Como valoración general de la mejora que la herramienta proporciona en el aula, se observó un incremento en la asistencia (sesiones en las que se usó Kahoot) y un mayor interés en la actividad de la claseen participar, factores que, sin duda alguna, contribuyen de manera favorable en el proceso de enseñanza y aprendizaje de las matemáticas en general.

\section{Referencias}

Guimares, D. (2015). Kahoot: quizzes, debates e sondagens. En A. A. A., Carvalho. Apps para dispositivos móveis: manual para professores, formadores e bibliotecários (pp. 203-224).: http://goo.gl/btf1G3

Guzmán, M. (1989). Juegos y matemáticas. Revista Suma, 4, 61-64. https://revistasuma.es/IMG/ pdf/4/061-064.pdf

Moya, M.M.; Carrasco, M. M.; Jiménez,M. A.; Ramón,A.; Soler,C.; Vaello,M. T. (2016). El aprendizaje basado en juegos: experiencias docentes en la aplicación de la plataforma virtual "Kahoot". Actas XIV Jornadas de Redes de Investigación en Docencia Universitaria. Alicante, 30 de junio y 1 de julio de 2016. https:// rua.ua.es/dspace/bitstream/10045/59136/1/ XIV-Jornadas-Redes-ICE 090.pdf

Muñoz, M. (2016). Las TIC en educación: "kahoot!" como propuesta de gamificación e innovación educativa para Educación Secundaria en Educación Física. Trabajo de grado de maestría. Universidad Internacional de la Rioja, España. https://www.researchgate.net/ publication/313863266

Ortiz, R. (2014). El juego como recurso didáctico en el aprendizaje de las matemáticas en alumnos de 6to grado de primaria. Trabajo de grado de Licenciatura en Educación. Universidad Pedagógica Nacional, México. http://200.23.113.51/pdf/30981.pdf
Parreño, N. (2017). Guía de estrategias lúdicas "jugando con los números" Para disminuir la discalculia en niños de sexto año de Educación Básica. Trabajo de grado de maestría. Universidad Nacional Chimborazo, Ecuador. http://dspace.unach.edu.ec/bitstream/51000/3370/2/ UNACH-EC-IP-EMIP-2017-0004.1.pdf

Ribosa, J.; Durán, D. (2017). Cooperación, juego y matemáticas: análisis de la aplicación del Tridio Cooperativo con alumnado de primaria. PNA, 11(3), 205-231.

Rodríguez, A. (2017). El juego como herramienta del aprendizaje del álgebra en las matemáticas de $3^{\circ}$ de eso. Trabajo de grado de máster. Universidad Internacional de la Rioja, España. https://reunir.unir.net/bitstream/ handle/123456789/4726/RODRIGUEZ \%20 GARCIA \%2C \%20ANA \%20ELENA. pdf? sequence $=1$ \&isAllowed $=y$

Rodríguez-Fernández, L. (2017). Smartphones y aprendizaje: el uso de Kahoot en el aula universitaria. Mediterranean Journal of Communication, 8(1), 181-190. https://doi.org/10.14198/ MEDCOM2017.8.1.13

Rodríguez, L.; Alonso, P.; Muñiz, L. (2014). El uso de los juegos como recurso didáctico para la enseñanza y el aprendizaje de las Matemáticas: estudio de una experiencia innovadora. Revista Iberoamericana de Educación Matemática, 39(2), 19-33. http://www.fisem.org/ www/union/revistas/2014/39/archivo6.pdf

Sánchez, G. (2010). Las estrategias de aprendizaje a través del componente lúdico. Marcoele. Revista de Didáctica Español como lengua extranjera,11, 1-66. https://marcoele.com/descargas/11/sanchez-estrategias-ludico.pdf

Valijas de herramientas TIC (2016). Kahoot! Guía de uso. http://valijas.ceibal.edu.uy/storage/ app/media/Guias\%20de\%20uso/Kahoot.pdf

Zarzycka, E. (2014). Kahootit or not? Can games be motivating in learning gramar? Teaching English with Technology, 16(3), 17-36. http:// goo.gl/OpchTz 